\title{
Spine neck plasticity regulates compartmentalization of synapses
}

Jan Tønnesen ${ }^{1,2}$, Gergely Katona ${ }^{3}$, Balázs Rózsa ${ }^{3}$, U. Valentin Nägerl ${ }^{1,2 *}$

\begin{abstract}
Affiliations:
${ }^{1}$ Interdisciplinary Institute for Neuroscience (IINS), Université Bordeaux Segalen, France

${ }^{2}$ UMR 5297, Centre National de la Recherche Scientifique (CNRS), Bordeaux, France

${ }^{3}$ 2-photon Imaging Center, Institute of Experimental Medicine of the Hungarian Academy of Sciences, Budapest, Hungary.

"Corresponding author: valentin.nagerl@u-bordeaux2.fr
\end{abstract}

Author contributions: Conceived and designed experiments (JT, VN), performed experimental work (JT), developed and provided reagents (GK, BR), analyzed data and performed modeling (JT, VN), wrote the paper (JT, VN), approved the paper (JT, GK, BR, VN). 


\begin{abstract}
Dendritic spines have been proposed to transform synaptic signals through chemical and electrical compartmentalization. However, the quantitative contribution of spine morphology to synapse compartmentalization and its dynamic regulation are still poorly understood.
\end{abstract}

We used time-lapse superresolution STED imaging in combination with FRAP measurements, 2photon glutamate uncaging, electrophysiology and simulations to investigate the dynamic link between nanoscale anatomy and compartmentalization in live spines of CA1 neurons in mouse brain slices.

We report a diversity of spine morphologies that argues against common categorization schemes, and establish a close link between compartmentalization and spine morphology, where spine neck width is the most critical morphological parameter. We demonstrate that spine necks are plastic structures that become wider and shorter after LTP. These morphological changes are predicted to lead to a substantial drop in spine head EPSP, while leaving overall biochemical compartmentalization preserved. 
Dendritic spines form the postsynaptic component of most excitatory synapses, whose plasticity is essential for brain development and higher brain functions ${ }^{1,2}$. In addition to the molecular composition of the synapse, the morphology of spines is thought to be critical for synaptic function, as spine head size correlates with synaptic strength ${ }^{3,4}$ and undergoes changes during synaptic plasticity ${ }^{5-8}$. Even so, our understanding of how spine structure shapes synapse function remains fragmented.

It is well established that spines compartmentalize biochemical signals ${ }^{9}$. By contrast, the quantitative contribution of spine morphology to compartmentalization is still unknown, and only moderate correlations between spine neck length or head volume and chemical diffusion have been reported ${ }^{9-11}$. It is an open question to what extent biochemical compartmentalization is determined primarily by spine geometry or intracellular factors such as organelles or protein assemblies.

Concerning electrical compartmentalization, it is not clear how electrical signals are transformed by the spine neck ${ }^{9,12-14}$. This is an important question because synaptic strength may be adjusted through structural changes in spine necks, which has been a long-standing hypothesis ${ }^{15,16}$.

An early electron microscopy study reported that the average spine head becomes larger and the neck wider and shorter after the induction of long-term plasticity (LTP) ${ }^{17}$, which was corroborated more recently by work based on 2-photon microscopy $y^{6,18,19}$. However, it is not known how these structural changes might affect biochemical and electrical compartmentalization, because 2-photon microscopy does not have sufficient spatial resolution to properly resolve spines and electron microscopy cannot be combined with functional assays. 
Here, we combined stimulated emission depletion (STED) microscopy, fluorescence recovery after photo-bleaching (FRAP) experiments, 2-photon glutamate uncaging, and patch-clamp electrophysiology in living cultured mouse brain slices and computer simulations, to directly relate spine morphological measurements to functional assays.

Our experiments clearly establish that spine morphology plays a determining role for biochemical and electrical compartmentalization, which can vary independently of each other. LTP leads to coordinated morphological changes in spine heads and necks, which leave overall biochemical compartmentalization intact, but are predicted to substantially impact EPSPs in potentiated spines. Furthermore, our study argues against common categorization schemes of spine morphology and indicates that stubby spines are substantially over-reported in the light microscopic literature due to limited spatial resolution.

\section{RESULTS}

\section{Quantitative analysis of spine morphological parameters}

We imaged spines on secondary and tertiary dendritic branches of CA1 pyramidal neurons by STED microscopy in 2 to 4 week old organotypic hippocampal slice cultures. The images revealed a continuum of morphologies (Fig. 1a; Supplementary Fig. 1a,b). Spine neck widths were symmetrically distributed around a median of $147 \mathrm{~nm}$, ranging from 51 to $279 \mathrm{~nm}(n=309$ spines [15 slices, 10 animals]; Fig. 1a-c; Table 1), while neck length was $667 \mathrm{~nm}$ and head width $519 \mathrm{~nm}$ (Fig. 1d,e; Table 1). The distributions of the morphological parameters appeared smooth and unimodal (Fig. 1c-e) and none of the morphological parameters were inter-correlated (Fig. 1f-h). 
We also imaged acute slices from 4 to 5 week old Thy1-YFP mice $(n=59$ spines $[4$ slices, 3 animals]). The ranges and distributions of morphological parameters were very similar between the two preparations (Fig. 1c-h; Table 1). In acute brain slices the median neck width was slightly larger than in organotypic slices (165 nm; Mann-Whitney test; $p=0.002$; Fig. 1c), which may be a genuine difference, or reflect a slight decrease in optical resolution due to the larger imaging depth ${ }^{20}$ (See Online Methods). In addition, there was a weak correlation between neck width and neck length in acute slices $\left(R^{2}=0.21\right.$; Fig. 1g). All remaining experiments were carried out in organotypic cultures.

The morphological parameters did not undergo directional changes (Kruskal-Wallis test $p>0.96$ for each of head width, neck width and neck length, respectively, over time; $n=33$ spines [2 slices, 2 animals]; Fig. 1i-l), indicating that spine morphology, especially spine neck length, is largely stable over periods of an hour (Fig. 1m) and that repeated STED imaging did not induce visible photo damage.

Surprisingly, we observed few, if any, stubby spines (lacking an identifiable neck) in the STED images, which is in contrast to the light microscopic literature that commonly reports high fractions of stubby spines (up to $40 \%{ }^{21-23}$ ). However, direct comparison between STED and 2photon images showed that short-necked spines frequently appear stubby in 2-photon mode, because of its lower optical resolution (Fig. 1n). Similarly, after spatially filtering the STED images to mimic the 2-photon case, short-necked spines wrongfully appear stubby (Fig. 1o). Moreover, given the limited $\mathrm{z}$ axis resolution of our STED approach, some spines might appear stubby because of a projection artifact in the $\mathrm{z}$ axis, even if the resolution in $\mathrm{x}-\mathrm{y}$ is very high. 
Taken together, the STED images reveal structural details and a diversity of spine shapes and sizes that validates previous electron microscopy studies ${ }^{24,25}$, and highlight the shortcomings of imaging spines with diffraction-limited resolution.

\section{Diffusional coupling of spines}

To assess the degree of biochemical compartmentalization of spines we performed FRAP experiments using freely diffusible YFP (27 KD) and Alexa 488 (0.64 KD). The recovery time course of the FRAP signal in individual spines could be well described by an exponential function with a single time constant $\tau$ (Fig. 2a; Suppl. Fig. 2), where $\tau$ reflects the degree of biochemical compartmentalization. The coefficient of variation of repeated $\tau$ measurements in individual YFP labeled spines was small $(22 \pm 6.7 \% ; 6$ repeated FRAP measurements in 11 spines, mean \pm standard deviation; Fig. 2b,c), indicating the approach is well suited for reporting differences between spines. In contrast, $\tau_{\text {yfp }}$ varied greatly from spine to spine (Fig. 2d), ranging from 42 ms to $2259 \mathrm{~ms}$ (median $=399 \mathrm{~ms} ; n=148$ spines [15 slices, 12 animals]; Fig. 2e). Alexa 488 has a 4.8 fold smaller hydrodynamic radius than YFP $(0.58 \mathrm{~nm} \text { versus } 2.8 \mathrm{~nm})^{26,27}$, and should diffuse accordingly faster if the diffusional milieu affects the two molecules similarly ${ }^{28}$. Indeed, fluorescence recovery was much faster for Alexa $488\left(\tau_{\text {alexa }}\right.$ median $=63 \mathrm{~ms} ; n=85$ spines [5 slices, 4 animals]; Fig. 2e) than for YFP. Like for YFP, $\tau_{\text {alexa }}$ values differed greatly between spines, ranging from $14 \mathrm{~ms}$ to $292 \mathrm{~ms}$ (Fig. 2e), while varied little within spines $(\mathrm{CV}=$ $18 \pm 4.7 \%$ (standard deviation)) from 6 repeated FRAP measurements in 9 spines; Fig. 2 b, c). 
The ratio of the median time constants $\tau_{\mathrm{yfp}} / \tau_{\text {alexa }}$ was 6.3 , close to the ratio of their hydrodynamic radii, suggesting that diffusion of molecules in and out of spines is largely determined by their size ${ }^{28}$.

\section{Spine morphology predicts synapse compartmentalization}

In contrast to previous studies, our superresolution-based approach allows for direct correlations between morphology and diffusion measurements in single spines. The applied correlation tests are justified by a simple compartmental model where the theoretically predicted $\tau$ is given by:

$$
\tau_{\text {calc }}=\frac{V * L}{D * A}
$$

[ $V$ is head volume, $L$ is neck length, $D$ is diffusion coefficient, $A$ is neck cross sectional area, and assuming $\left.D_{\text {yfp }}=16 \mu \mathrm{m}^{2} / \mathrm{s}^{29}, D_{\text {alexa }}=120 \mu \mathrm{m}^{2} / \mathrm{s}^{30}\right]$.

Plotting $\tau_{\text {yfp }}$ as a function of spine neck width revealed a strong negative correlation, well described by an inverse square function based on Eq. 1, $\left(\mathrm{y}=\mathrm{ax}^{-2}+\mathrm{b} ; R^{2}=0.47 ; n=148\right.$; Fig. 3a,b), indicating that neck width is a determining factor of $\tau_{\text {yfp }}$ and that small differences in spine neck diameter have large effects on chemical diffusion. Additionally, there was a positive correlation between head width and $\tau_{\mathrm{yfp}}$, described by a cubic fit $\left(y=a x^{3}+b\right)$ with a moderate to strong correlation $\left(R^{2}=0.33\right.$; Fig. 3c), while neck length was a weaker predictor of $\tau_{\text {yfp }}$ (using a linear fit function; $R^{2}=0.18$; Fig. 3d). Similarly, $\tau_{\text {alexa }}$ depended moderately to strongly on spine neck width $\left(R^{2}=0.31 ; n=85\right.$; Fig. 3e,f), corroborating that biochemical compartmentalization depends sensitively on spine neck geometry. 
When normalizing the Alexa 488 diffusion data by the factor 6.3 (the ratio of medians $\tau_{\mathrm{yfp}} /$ $\tau_{\text {alexa }}$ ), we found no differences between the diffusion of YFP and Alexa 488 for given neck width bins (Sidak's multiple comparisons, all non-significant; Fig. 3g).

Based on linear regression analysis, neck length explained $18 \%$ of the variation in $\tau_{\text {yfp }}($ Fig. 3d), neck cross sectional area explained $45 \%\left(R^{2}=0.45\right)$, and head volume $27 \%$, while the respective residual plots validated the use of linear fits (Suppl. Fig. 3b-e). Overall, 60\% of the variation in $\tau_{\text {yfp }}$ could be explained by morphology (Fig. $\left.3 \mathbf{h}\right)$.

Taken together, the experiments established a strong link between spine morphology and biochemical compartmentalization, identifying spine neck width as the dominant parameter of the diffusional barrier.

\section{Estimating the electrical resistance of the spine neck}

The electrical resistance of the spine neck $\left(R_{\text {neck }}\right)$ can be estimated based on neck morphology or on spine head diffusion measurements using Ohm's or Fick's law, respectively9:

$R_{m o r p h}=\frac{\rho * L}{A}$

$R_{\tau}=\frac{\tau * \rho * D}{V}$

[ $\rho$ is resistivity of the cytoplasm, $D$ diffusion coefficient, $L$ spine neck length, $A$ neck cross section area, $V$ head volume and, and assuming $\rho=150 \Omega * \mathrm{~cm}^{31,32}, D_{\text {yfp }}=16 \mu \mathrm{m}^{2} / \mathrm{s}^{29}, D_{\text {alexa }}=$ $\left.120 \mu \mathrm{m}^{2} / \mathrm{s}^{30}\right]$. 
From these equations we calculated $R_{\text {neck }}$ in three independent ways, based either on morphology or diffusion measurements of YFP and Alexa 488, yielding highly consistent values and ranges centered around $56 \mathrm{M} \Omega\left(R_{\mathrm{morph}}=44[18,112] \mathrm{M} \Omega, n=148 ; R_{\tau \mathrm{yfp}}=62[35,98] \mathrm{M} \Omega, N=148 ; R_{\tau}\right.$ alexa $=57[27,108] \mathrm{M} \Omega, n=85$; median [inter-quartile range]; 1-way ANOVA, $p=0.07 ;$ Fig. $4 a, b)$.

Plotting the measured $\tau$ against the estimated $R_{\text {neck }}$ revealed a significant correlation for a majority of spines. Interestingly, an iterative approach found that it was absent in the population of spines with high $R_{\text {neck }}$ values, both for YFP $\left(R^{2}=0.28, p<0.001\right.$ for $R_{\text {neck }}<84 \mathrm{M} \Omega, n=100$ spines; in contrast $R^{2}=0.07, p=0.07$ for $R_{\text {neck }}>84 \mathrm{M} \Omega, n=48$ spines; Fig. 4c) and for Alexa $488\left(R^{2}=0.30, p<0.001\right.$ for $R_{\text {neck }}<80 \mathrm{M} \Omega, n=59$ spines; in contrast $R^{2}=0.14, p=0.06$ for $R_{\text {neck }}>80 \mathrm{M} \Omega, n=26$ spines; Fig. 4d). This de-correlation indicated that in highly compartmentalized spines, biochemical compartmentalization and electrical neck resistance vary independently of each other.

\section{Correlation between changes in morphology and diffusion}

To investigate how changes in morphology and diffusion co-vary at the level of individual spines, we whole-cell patch clamped CA1 neurons and depolarized them to $0 \mathrm{mV}$ for $4 \mathrm{~min}$, a protocol reported to decrease diffusional coupling ${ }^{33}$.

Indeed, this manipulation increased $\tau_{\text {alexa }}$ by $36 \pm 12 \%$ (mean \pm CI; Wilcoxon paired test, $p=$ $0.001 ; n=25$ spines, Fig. 5a), while spine neck width decreased by just $6 \pm 2 \%$ (mean $\pm \mathrm{CI}$; paired t-test, $p=0.009 ; n=25$ spines; Fig. 5b), corresponding to a change in cross sectional area of $12 \%$. Even though both effects are modest, the changes in $\tau_{\text {alexa }}$ and neck width co-vary 
strongly $\left(R^{2}=0.34\right.$; Fig. $\left.\mathbf{5 c}\right)$, corroborating that molecular diffusion is very sensitive to changes in spine neck width.

Control experiments showed that voltage-clamping CA1 neurons at $-70 \mathrm{mV}$ by itself neither led to changes in neck width (mean $\pm \mathrm{CI} \Delta$ neck width $7 \pm 4 \%$, paired t-test; $p=0.16 ; n=12$ spines; Fig. 5d) nor $\tau_{\text {alexa }}$ (mean $\Delta \tau_{\text {alexa }} 4 \pm 3 \% ; p=0.62$; Fig. 5e). The depolarization protocol did not affect head width, head length and neck length (Paired t-tests; $p=0.26$ to 0.77 ; Fig. 5f).

\section{LTP leads to plasticity in spine head and neck geometry}

Next, we examined how spine morphology changes during spine-specific LTP ${ }^{6}$.

We measured excitatory postsynaptic currents in response to glutamate uncaging (uEPSCs) in the whole-cell patch-clamp configuration up to 60 min after 2-photon uncaging by short pulses $(1 \mathrm{~ms}, 0.5 \mathrm{~Hz}$ for $60 \mathrm{sec})$ in $\mathrm{Mg}^{2+}$ free solution. Similar to previous reports ${ }^{13,14}$, this protocol potentiated targeted spines by around $75 \%$ for at least 60 min (1-way ANOVA, $p=0.0008$, Dunnett's Multiple Comparison test, all time points after potentiation $p<0.05 ; n=10$ spines [8 slices, 3 animals]; Fig. 6a,b). In contrast, neighboring spines (within $5 \mu \mathrm{m}$ ) did not undergo potentiation (1-way ANOVA, $p=0.99, n=8$ spines [7 slices, 3 animals]; Fig. 6a,b). Applying the uncaging LTP ( $\mathrm{uLTP}$ ) protocol in the presence of $\mathrm{Mg}^{2+}$, where NMDA receptors remain blocked, failed to induce potentiation (1-way ANOVA, $p=0.99, n=6$ spines [5 slices, 3 animals]; Fig. 6a,b). 
Given that potentiation of uEPSCs could be reliably induced by this protocol (10 out of 11 spines responded with potentiation), we performed time-lapse STED imaging on unperturbed neurons, without electrophysiological recordings.

Induction of uLTP led to a large increase in head volume ( $390 \pm 11 \%$ (mean \pm s.e.m.), $n=16$ spines [12 slices, 6 animals]; Fig. 6c-f), followed by a stable plateau (around 160\% above baseline $)^{6,34}$. The changes were highly significant relative to before uncaging and to neighboring spines $\left(n=18\right.$ spines [12 slices, 6 animals]) and to uncaging on spines in the presence of $\operatorname{Mg}^{2+}(n$ $=9$ spines [6 slices, 4 animals]), none of which underwent head size increases (2-way ANOVA, effect pre versus post uncaging $p=0.0002$, effect uLTP versus neighbors versus $\mathrm{ULTP}+\mathrm{Mg}^{2+} p<$ 0.001; followed by Tukey's Multiple Comparisons Test all uLTP time points after uncaging $p<$ 0.01, all neighbors and $\mathrm{uLTP}+\mathrm{Mg}^{2}$ time points non-significant; Fig. 6f).

Concurrently, there was a highly significant decrease in neck length by around $25 \%$ in potentiated spines (2-way ANOVA, effect pre versus post uncaging $p=0.07$, effect of uLTP versus neighbor versus $\mathrm{uLTP}+\mathrm{Mg}^{2}$ spines $p=0.01$; Tukey's Multiple Comparisons Test uLTP all time points after uncaging $p<0.05$, all neighbors and $\mathrm{uLTP}+\mathrm{Mg}^{2}$ time points non-significant; Fig. 6g). Moreover, uLTP induction led to a sustained widening of spine necks by around $30 \%$ (2-way ANOVA, effect pre versus post uncaging 0.009, effect of uLTP versus neighbor versus $\mathrm{uLTP}+\mathrm{Mg}^{2}$ spines $p=0.02$; Tukey's Multiple Comparisons Test uLTP all time points after uncaging $p<0.05$, all neighbors and $\mathrm{uLTP}+\mathrm{Mg}^{2}$ time points non-significant; Fig. 6h).

\section{Impact on biochemical and electrical compartmentalization}


To understand the impact of these morphological changes (summarized in Suppl. Fig. 4) on synapse compartmentalization, we calculated $R_{\text {neck }}$ and $\tau$ during uLTP. On average $R_{\text {neck }}$ drops by around 50\% after uLTP (1-way ANOVA $p<0.0001$, Dunnett's Multiple Comparisons Test, all time points after uLTP $p<0.01$; Fig. 6i), whereas $\tau$ is predicted not to change significantly (1way ANOVA $p=0.28$; Dunnett's Multiple Comparisons Test, all time points after uLTP $p>$ 0.05; Fig. 6j). The surprising prediction that $\tau$ is preserved after LTP was confirmed by separate experiments (Fig. 6j, Suppl. Fig. 5). These experiments corroborate a strong correlation between measured and calculated $\tau$ in a dynamic setting $\left(R^{2}=0.49, n=41 \tau\right.$ recordings, $\mathrm{n}=13$ spines [6 slices, 4 animals]; Fig. $\mathbf{6 j}, \mathbf{k}$ ). Moreover, we found a strong negative correlation between changes in head volume and $R_{\text {neck }}$ after uLTP $\left(R^{2}=0.54\right.$; Fig. 61), indicating that these structural changes are coordinated.

Taken together, uLTP induction triggers changes in spine morphology that impact synapse compartmentalization in a complex way, leading to substantially increased electrical coupling while biochemical compartmentalization is largely preserved.

\section{Predicted effects of $\boldsymbol{R}_{\text {neck }}$ changes on EPSPs}

To explore the functional consequences of such changes in $R_{\text {neck }}$ we used a simplified electrical equivalent circuit of a passive spine, i.e. without voltage-dependent conductances ${ }^{13}$ (Suppl. Fig. 6a). We calculated EPSP amplitudes in the spine and dendrite for a physiologically relevant range of synaptic and dendritic parameters using the following equations:

$$
\operatorname{EPSP}_{\text {spine }}=\frac{G_{\text {syn }} * R_{\text {spine }} * E_{\text {syn }}}{1+G_{\text {syn }} * R_{\text {spine }}}
$$




$$
\operatorname{EPSP}_{\mathrm{dend}}=\frac{G_{\mathrm{syn}} * R_{\mathrm{dend}} * E_{\mathrm{syn}}}{1+G_{\mathrm{syn}} * R_{\mathrm{spine}}}
$$

[ $R_{\text {dend }}$ is the input resistance of the dendrite (assumed to be $50 \mathrm{M} \Omega^{35,36}$ ), $E_{\mathrm{syn}}$ is the synaptic reversal potential relative to the resting membrane potential of $-70 \mathrm{mV}, G_{\mathrm{syn}}$ the synaptic conductance and $\left.R_{\text {spine }}=R_{\text {neck }}+R_{\text {dend }}\right]$.

Because the capacitance of the spine head and neck membrane is negligibly small $(\sim 0.01 \mathrm{pF})$, it is not included in the model ${ }^{13}$.

The amplitudes of the EPSP in the spine head and the dendrite both depend strongly and positively on $G_{\text {syn }}(\mathbf{F i g}$. 7a,, $\mathbf{b})$, but they depend on $R_{\text {neck }}$ in opposite ways in that the EPSP in the dendrite gets attenuated, while in the spine it gets boosted with increasing resistance (Fig. 7c). To illustrate this effect in relative terms, we plotted the ratios of the spine and dendritic voltages as a function of $R_{\text {neck }}($ Fig. $7 \mathbf{c})$. Notably, the ratios depend solely on $R_{\text {neck }}$ and $R_{\text {dend }}$ and are independent of $G_{\mathrm{syn}}$ and $E_{\mathrm{syn}}{ }^{13}$.

To predict the effect of a $50 \%$ drop in $R_{\text {neck, }}$, we plotted the expected percentage changes of

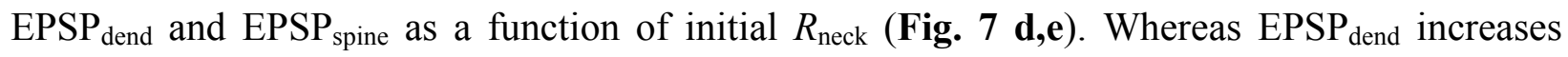
merely by a few $\%, \mathrm{EPSP}_{\text {spine }}$ is consistently reduced by 20 to $40 \%$ over a wide range of $G_{\text {syn }}$ and initial $R_{\text {neck }}$ values, indicating that physiological changes in spine neck geometry can substantially suppress the boosting of spine head voltages, but increase dendritic voltages only modestly (Suppl. Fig. 6b). These simulated effects on EPSPs solely reflect the observed changes in spine neck geometry, and do not take into account any increases in synaptic conductance after LTP. 


\section{DISCUSSION}

We performed time lapse imaging of dendritic spines in living brain slices with a lateral resolution around $50 \mathrm{~nm}^{37}$, in parallel with functional assays, effectively bridging the gap between electron microscopy and conventional fluorescence microscopy.

\section{Resolving live spine morphology}

Electron microscopy studies have provided exquisitely detailed and quantitative analyses of spine morphology in fixed samples ${ }^{24,38,39}$, but a comparable analysis in live tissue has been lacking. Our STED images reveal a high degree of heterogeneity of spine sizes and morphologies, which agrees well with the previous electron microscopy work, but argues against morphological categorization schemes commonly used in the light microscopic literature ${ }^{40-42}$.

Notably, our study indicates that stubby spines are greatly over-reported in the light-microscopic literature, due to insufficient optical resolution. This conclusion is supported by electron microscopy studies that typically observe only low fractions (a few percent) of stubby spines in adult tissue $24,25,38,43$. This is not merely a semantic issue because spines with large heads and thin and short necks (e.g. Fig. 1o) represent completely different functional compartments than spines devoid of necks.

\section{Morphology determines biochemical compartmentalization}

Synaptic strength can be regulated independently of neighboring synapses by way of compartmentalized signaling ${ }^{6,34}$, which is thought to boost the computational power of neurons. 
We found that $60 \%$ of the variation in biochemical compartmentalization across spines could be accounted for by morphology, and that changes in spine structure strongly impact molecular diffusion. Neck width was the most influential determinant of compartmentalization, potentially facilitating fast and cost-efficient regulation of the synaptic milieu. However, we cannot rule out that intracellular factors, such as organelles, co-vary with the morphology and also play a role. Even so, nanoscale spine structure can be used as a reliable proxy for synapse compartmentalization.

The finding that fluorescence recovery of Alexa 488 and YFP largely followed basic diffusion theory suggests that the diffusion barrier holds for a wide range of signaling molecules, e.g. $\mathrm{Ca}^{2+}$, cAMP and $\mathrm{IP}_{3}$, and larger cytosolic proteins, e.g. GTPases, actin or monomeric CaMKII. Still, the slightly higher than expected median $\tau$ ratio (relative to the ratio of the hydrodynamic radius) may reflect a modest sieving effect, which slows down the diffusion of molecules based on their size.

\section{Reliable estimates of spine neck resistance}

As electrical measurements of $R_{\text {neck }}$ are technically not possible, we estimated $R_{\text {neck }}$ in three different indirect ways, using basic biophysical equations and the morphological and diffusional data. The fact that the independent estimates are highly consistent suggests that the measurements were robust and accurate.

Previous studies based on $\mathrm{EM}^{25}$ or diffusion measurements ${ }^{9}$ reported a range of 1 to $400 \mathrm{M} \Omega$ for $R_{\text {neck, }}$, whereas a recent study based on $\mathrm{Ca}^{2+}$ imaging estimated $R_{\text {neck }}$ to be relatively large (500 $\mathrm{M} \Omega$ ) and to very little across spines, which suggests that morphology does not a play a major 
role $^{44}$. Encompassing these values, our measurements revealed a broad distribution, indicating that, at any given time, half of all spines have $R_{\text {neck }}$ values larger than $56 \mathrm{M} \Omega$, with $5 \%$ having resistances larger than $500 \mathrm{M} \Omega$. The previous discrepancies may be due to relatively low $\mathrm{N}$ in some reports and/or biases inherent to the methods that were used, which may have compressed biological variability.

\section{LTP leads to coordinated changes in spine heads and necks}

A multitude of diverging observations regarding spine neck plasticity can be found in the existing literature ${ }^{45}$. Whereas spine head enlargement after the induction of LTP has been consistently reported ${ }^{5,6,19,34}$, much less is known about changes occurring at the level of spine necks.

Consistent with our observations, an early electron microscopy study reported changes in average spine neck geometry after tetanic stimulation ${ }^{17}$. However, as electron microscopy cannot be used for longitudinal investigations, the reported differences between the stimulated and control groups may have been due to altered turnover of populations of spines of certain sizes, as opposed to reflecting dynamic changes at the level of individual spines. A recent 2-photon study reported changes in spine neck fluorescence, which were interpreted as shortening and widening after $\mathrm{LTP}^{19}$, however, the quantification is problematic because of the limited optical resolution.

Here, we present direct and clear evidence based on time lapse STED imaging that spine necks are highly plastic structures, becoming substantially shorter and wider in a spine-specific LTP paradigm. In addition, the analysis shows that structural changes in spine necks and heads occur 
in a concerted fashion, with spine head enlargement scaling with neck shortening and broadening.

\section{Functional impact of spine neck plasticity}

Our data indicate that the observed morphological changes have diverging consequences for biochemical and electrical compartmentalization, which is theoretically possible given that $\tau$ depends on neck and spine head size (Eq. 1), whereas $R_{\text {neck }}$ depends only on neck size (Eq. 2).

Whereas spine head enlargement increases biochemical compartmentalization, the observed neck changes counteract this effect, so that diffusional recovery is largely preserved after LTP. Nevertheless shorter and wider necks may facilitate access to the spine from the dendritic side ${ }^{46}$, and chemical second messengers released into the enlarged spine head might be more diluted and disperse faster into the dendrite, which may render spines less susceptible to subsequent plasticity events.

In contrast to biochemical compartmentalization, our simulations predict electrical signalling to be substantially altered by spine neck plasticity. While a $50 \%$ drop in $R_{\text {neck }}$ is predicted to lead to only a slight increase in dendritic EPSPs, the amplitude of the EPSP in the spine would drop by $20 \%$ to $40 \%$ in the majority of spines.

With substantially reduced boosting of the spine head voltage after a sharp drop in $R_{\text {neck, }}$, synapses will operate in a more linear regime: voltage-gated channels and NMDA receptors are less likely to get activated ${ }^{47}$, and the voltage in the spine head is less likely to reach the synaptic reversal potential and saturate the synaptic response ${ }^{48}$. Spines with shorter and wider necks will 
be able to sustain stronger synaptic currents, because the driving force will be effectively maintained even during large or repeated synaptic conductance changes. In this way, the observed neck changes may functionally disinhibit the synapse, which could contribute to synaptic weight changes during LTP ${ }^{44,49}$.

Taken together, our findings challenge the widespread notion that spines primarily shape biochemical rather than electrical signaling at synapses. Instead, they argue that both functions are distinctly shaped and dynamically regulated by nanoscale spine morphology.

\section{ACKNOWLEDGEMENTS}

We thank D. DiGregorio (Pasteur Institute) and all members of the Nägerl lab for comments on the manuscript, and J. Angibaud for excellent technical support. This work was supported by consecutive postdoctoral fellowships from the Marie-Curie Program (Grant 272351) and EMBO (Grant 1518-2010) to JT, and grants from Inserm, ANR and HFSP (UVN). 
Table 1. Results

\begin{tabular}{lccccc}
\hline Organotypics & Range & Mean & Median & Quartile range & $\begin{array}{c}\text { n (spines/ } \\
\text { slices/animals) }\end{array}$ \\
Neck width & 51 to 279 & 150 & 147 & 124 to 173 & $309 / 15 / 10$ \\
Neck length & 70 to 2368 & 743 & 667 & 397 to 1037 & $309 / 15 / 10$ \\
Head width & 190 to 1482 & 575 & 519 & 385 to 730 & $307 / 15 / 10$ \\
\hline Acute slices & & & & & \\
Neck width & 59 to 292 & 167 & 165 & 147 to 188 & $59 / 4 / 3$ \\
Neck length & 157 to 1801 & 689 & 551 & 260 to 993 & $59 / 4 / 3$ \\
Head width & 262 to 1104 & 583 & 564 & 397 to 756 & $59 / 4 / 3$ \\
\hline $\boldsymbol{\tau}$ (ms) & & & & & \\
$\tau_{\text {yfp }}$ & 42 to 2259 & 518 & 399 & 206 to 676 & $148 / 15 / 12$ \\
$\tau_{\text {alexa }}$ & 14 to 292 & 80 & 63 & 36 to 105 & $85 / 5 / 4$ \\
\hline $\boldsymbol{R}_{\text {neck }}$ (M) & & & & & \\
$R_{\text {morph }}$ & 2.1 to 598 & 82 & 44 & 18 to 113 & $148 / 15 / 12$ \\
$R_{\tau \text { tyfp }}$ & 9.2 to 807 & 93 & 62 & 35 to 98 & $148 / 15 / 12$ \\
$R_{\tau \text { alexa }}$ & 4.9 to 1263 & 115 & 57 & 27 to 108 & $85 / 5 / 4$
\end{tabular}




\section{FIGURE LEGENDS}

\section{Figure 1. Spine morphologies span a broad continuum}

(a) STED images of dendritic segment. The image is a maximum intensity projection of $10 \mathrm{z}$ planes $460 \mathrm{~nm}$ apart (raw image in Suppl. Figure 1). (b) Zoom in on spine of box insert in (a). The intensity profile of neck is depicted (yellow) with a Gaussian fit (dotted red), the full width at half maximum (FWHM; blue line) indicate neck width. (c) Distribution of neck width measurements in organotypic cultures. (d) Distribution of neck length measurements in organotypic cultures. (e) Head width distribution. Inserts in (c-e) show median, inter-quartile and range, see also Table 1. (c-e) Distribution of morphological parameters of spine in acute slices. Curved lines are log-normal fits where $R^{2}=0.92$ to 0.97 for organotypic data and $R^{2}=0.72$ to 0.85 for acute slice data. (f) Linear correlations between neck and head widths, (g) neck width and neck length, (h) neck length and head width. (i) Time-lapse imaging over 1 hour (geometric mean with $95 \% \mathrm{CI}),(\mathbf{j}-\mathbf{l})$ Standard deviation of the normalized changes (corresponding to the coefficient of variation). (m) Variability of morphological parameters over one hour $(p=0.03$; mean \pm s.e.m.). (n) Spines appear stubby in 2-photon, but not in STED mode (red arrows). (o) Degrading a spine STED image digitally by convolving with a $200 \mathrm{~nm}$ Gaussian function makes the highlighted spine (blue arrow) appear stubby. All scale bars represent $500 \mathrm{~nm}$.

\section{Figure 2. Spines are heterogeneous biochemical compartments}

(a) Example of FRAP trace with mono-exponential fit (red), yielding the time constant $\tau$. The intensity bar is relative fluorescence of the line scan. (b) Variability of repeated FRAP measurements within spines. Scale bars are $250 \mathrm{~ms}$. (c) mean CVs ( \pm standard deviation) for 
YFP and for Alexa 488. Variability was not different between the two fluorophores $(p=0.15)$.

(d) Variability in compartmentalization between spines. The traces were recorded at the colorcoded lines in the three spines. The bottom trace (dark lilac) shows bleaching and recovery in the spine head, while the flat trace (light lilac) shows the fluorescence in the dendrite. Scale bars represent $500 \mathrm{~nm}$ for spines and $1 s$ for FRAP traces. (e) Range and distribution of all YFP and Alexa 488 FRAP measurements. Scatter plots show all data points while the box plots show the median, inter-quartiles and range.

\section{Figure 3. Spine morphology determines compartmentalization}

(a) Two examples of YFP labeled spines and their corresponding FRAP traces and $\tau$ values. Neck diameters are indicated. Scale bars represent $500 \mathrm{~nm}$ for spines (applies to all spines) and $500 \mathrm{~ms}$ for FRAP traces. (b) $\tau$ plotted as a function of neck width. The inverse square fit is shown with the $95 \%$ confidence interval. (c) $\tau$ plotted as a function of head width (cubic fit). (d) $\tau$ plotted as a function of neck length. Linear regression with residuals between $\tau$ and neck crosssectional area, head volume or neck length is shown in Supplementary Figure 3. (e) Two examples of Alexa 488 labeled spines and recovery traces. Red scale bar is $250 \mathrm{~ms}$. (f) $\tau_{\text {alexa }}$ as a function of neck width. (g) $\tau_{\text {yfp }}$ and $\tau_{\text {alexa }}$ plotted relative to binned neck widths (Normalized bin heights not different; Sidak's multiple comparisons, all $p>0.05)$. The two axes are scaled by a factor of 6.3, which equals the ratio median $\tau$ of YFP over Alexa. Bars indicate mean \pm standard deviation. (h) Calculated $\tau$ (Eq. 1) plotted against measured $\tau$. 


\section{Figure 4. Estimating electrical neck resistance}

(a) Distributions of estimates of $R_{\text {neck }}$ based on morphology or diffusion (YFP and Alexa 488) measurements, which did not significantly differ $(p=0.07$; plot depicts median, quartiles and range). (b) Cumulative probability plot of all spine neck resistances with median $56 \mathrm{M} \Omega$. (c) $\tau_{\mathrm{yfp}}$ as a function of $R_{\text {neck }}$ (overall fit not shown, $R^{2}=0.32$ ). For values of $R_{\text {neck }}$ larger than $84 \mathrm{M} \Omega$ (dotted line) the correlation was non-significant $\left(R^{2}=0.07, p=0.07, n=48\right.$ spines), while it was moderate below this value $\left(R^{2}=0.28, p<0.001, n=100\right)$. (d) The same observation was made for $\tau_{\text {alexa, }}$, where the correlation was insignificant above $80 \mathrm{M} \Omega$ (dotted line; $n=26$ spines; $p=$ $\left.0.06, R^{2}=0.14\right)$, while highly significant below, with a moderate correlation $(n=59$ spines; $p<$ $\left.0.0001, R^{2}=0.30\right)$.

\section{Figure 5. Changes in diffusion and spine neck width co-vary}

(a) Change in $\tau_{\text {alexa }}$ after a 4 min depolarization to $0 \mathrm{mV}$. (b) Effect of depolarization on the spine neck width. (c) Changes in $\tau_{\text {alexa }}$ as a function of neck width change (Depicted with $95 \%$ CI. (d) $\tau_{\text {alexa }}$ of control cells, which were kept at $-70 \mathrm{mV}$. (e) Neck width changes of control cells measured at two time points $5 \mathrm{~min}$ apart. (f) Depolarization did not lead to changes in head width, head length and neck length ( $p=0.26$ to 0.77$)$. Before/after plots depict mean $\pm 95 \%$ CI.

\section{Figure 6. Structural neck and head plasticity during LTP}

(a) Uncaging EPSCs were measured before and up to 60 min after LTP induction. (b) Effect of the uncaging LTP protocol on EPSCs (Graph shows mean change \pm s.e.m.) from targeted spines, neighboring spines within $5 \mu \mathrm{m}$, and spines subjected to the uLTP protocol in the presence of 
$\mathrm{Mg}^{2+}$. Morphological changes during LTP were assessed in a separate set of neurons that were not patch-clamped. (c) Effect of uLTP targeting the spine identified by the arrow. (d) Another example uLTP induced changes in morphology in a potentiated spine (boxed). (e) Zoom of the boxed spine in (d). (f) Effect of LTP on head size (mean \pm s.e.m.). (g) Effect of uLTP on neck length, (h) and neck width. (i) Calculated changes in $R_{\text {neck }}$ and $\tau$ from the morphological measurements during LTP. (j) Calculated and observed changes in $\tau$ after uLTP (The corresponding morphological changes are depicted in Suppl. Fig. 5). (k) Pair-wise comparison of changes in observed and calculated $\tau$ during LTP depicted in $(\mathbf{j}) .(\mathbf{m})$ Correlation of the initial head volume change to the corresponding $R_{\text {neck }}$ change (at $1 \mathrm{~min}$; data from (f-g)). Time lapse graphs display mean with s.e.m., and correlations plots are depicted with the $95 \%$ CI. Asterisks denote $* p<0.05 ; * * p<0.01 ; * * * p<0.001$.

\section{Figure 7. Influence of $\boldsymbol{R}_{\text {neck }}$ on electrical signaling}

(a) Simulation of the dependence of the dendritic EPSP on $R_{\text {neck }}$ for three values of synaptic cunductances $\left(G_{\mathrm{syn}}\right)$. The $56 \mathrm{M} \Omega$ median $R_{\text {neck }}$ is depicted as a dotted line. (b) The corresponding EPSPs in the spine head as a function of $R_{\text {neck. }}$ (c) Ratios EPSP $_{\text {spine }} /$ EPSP $_{\text {dend }}$ and $\mathrm{EPSP}_{\text {dend. }} / \mathrm{EPSP}_{\text {spine }}$ relative to $R_{\text {neck. }}$ The ratios are independent of $G_{\text {syn. }}$ (d) Percentage change in dendritic EPSP caused by a $50 \%$ drop in $R_{\text {neck, }}$ as a function of the initial $R_{\text {neck. }}$ (e) The corresponding EPSP change in the spine head. 


\section{ONLINE METHODS}

\section{Animals}

All experiments were performed in organotypic or acute hippocampal brain slices from Thy1YFP-H transgenic mice, in which a subset of hippocampal CA1 pyramidal neurons strongly expresses YFP (Jackson Labs, Bar Harbor, ME). Experimental procedures were in accordance with the French National Code of Ethics on Animal Experimentation and approved by the Committee of Ethics of Bordeaux (No. 50120202).

\section{Organotypic slice cultures and acute slices}

Organotypic hippocampal slice cultures (Gähwiler type) were dissected from 5-7 day pups of Thy1-YFP transgenic mice and cultured 2 to 4 weeks at $35^{\circ} \mathrm{C}$ in a roller drum at 10 revolutions per hour as previously described ${ }^{50,51}$. In brief, hippocampi were chopped coronally at $350 \mu \mathrm{m}$ using a tissue chopper and embedded in a freshly mixed plasma/thrombin clot on the surface of a poly-L-lysine (PLL) coated glass coverslip. After coagulation, the slice on the coverslip is cultured in a roller tube in $0.5 \mathrm{ml}$ of medium consisting of 50\% Eagle's Basal Medium, 25\% horse serum and 25\% Hank's Balanced Salt Solution, supplemented with Glutamine to a final concentration of $1 \mathrm{mM}$ and Glucose $(11 \mathrm{~g} / \mathrm{L})$ (all from Sigma). The Gähwiler cultures are optically very accessible, as synapses close to the coverslip can be imaged on an inverted microscope setup.

Acute hippocampal slices from 4 to 5 week old Thy1-YFP-H mice were cut on a vibratome at $350 \mu \mathrm{m}$ in chilled solution consisting of $195 \mathrm{mM}$ Sucrose, $2.5 \mathrm{mM} \mathrm{KCl}, 1.25 \mathrm{mM} \mathrm{NaH}_{2} \mathrm{PO}_{4}, 28$ 
$\mathrm{mM} \mathrm{NaHCO} 3,0.5 \mathrm{mM} \mathrm{CaCl}_{2}, 1 \mathrm{mM}$ ascorbic acid, $7 \mathrm{mM}$ glucose and $7 \mathrm{mM} \mathrm{MgCl}_{2}$. After cutting, slices were allowed to recover for an hour. Immediately before imaging, slices were placed on PLL coated glass coverslips, to which they would adhere, and transferred to the perfused imaging chamber.

\section{STED microscope and imaging}

Superresolved images of spines were acquired by STED microscopy ${ }^{52,53}$. The home-built STED microscope was described previously ${ }^{37}$. Briefly, it is constructed around an inverted confocal microscope (DMI6000, Leica) using a 100x, 1.4 NA oil immersion objective (PL APO 100, Leica). It uses pulsed excitation at $488 \mathrm{~nm}$ (PicoQuant) and pulsed quenching at $594 \mathrm{~nm}$. The quenching wavelength is derived from an optical parametric oscillator (OPO) pumped at $796 \mathrm{~nm}$ by a Ti:Sa femtosecond laser (MaiTai, SpectraPhysics). Emitted fluorescence is detected by an avalanche photo-diode (APD, Perkin Elmer). Pixel dwell times were 40 to $50 \mu$ s for STED images, and pixel size was typically $20 \mathrm{~nm}$ by $20 \mathrm{~nm}$. Typically three to five z-sections were acquired $400 \mathrm{~nm}$ to $500 \mathrm{~nm}$ apart. The effective lateral optical resolution of this setup is around $50 \mathrm{~nm}$ for work in live organotypic slices ${ }^{37}$.

A second Ti:Sa pulsed femtosecond laser (MaiTai, SpectraPhysics) in the infra-red range was routed in and co-aligned with the excitation/quenching beams. This beam was used for 2-photon mediated bleaching of YFP at $900 \mathrm{~nm}$ and Alexa Fluor 488 at $800 \mathrm{~nm}$, as well as for 2-photon imaging at $900 \mathrm{~nm}$ and 2-photon glutamate uncaging at $740 \mathrm{~nm}$. Image acquisition was done

using ImSpector software ${ }^{54}$. Experiments were performed in artificial cerebro-spinal fluid containing $125 \mathrm{mM} \mathrm{NaCl}, 2.5 \mathrm{mM} \mathrm{KCl}, 1.3 \mathrm{mM} \mathrm{MgCl}_{2}, 2 \mathrm{mM} \mathrm{CaCl}_{2}, 26 \mathrm{mM} \mathrm{NaHCO} 3,1.25$ $\mathrm{mM} \mathrm{NaH}{ }_{2} \mathrm{PO}_{4}, 20 \mathrm{mM}$ D-glucose, $1 \mathrm{mM}$ Trolox; $300 \mathrm{mOsm}$; pH 7.4. Perfusion rate was 2 
$\mathrm{ml} / \mathrm{min}$ and the temperature $31.5^{\circ} \mathrm{C}$. Since our STED approach enhanced resolution only in the lateral plane, we limited our analysis to spines extending laterally from the dendrite. For imaging of acute slices the microscope objective was changed to a glycerin objective (NA 1.3) equipped with a correction collar (Leica), which facilitates superresolution imaging at tens of microns tissue depth, which is not possible with oil objectives (NA 1.4) because of spherical aberrations ${ }^{55}$. However, because of the lower numerical aperture, the achievable resolution is slightly lower than with oil.

Geometric measurements of morphological parameters were done on raw images of single sections in ImageJ. Spine neck widths were obtained from full width half-maximum (FWHM) measurements based on Gaussian fits of line profile plots as $i^{56}$. Each line profile was obtained from a 3 pixel wide line, to avoid noisy single pixel outliers. Neck length was measured from the base of the parent dendrite to the base of the head, following the curvature of the spine neck. Head width is measured perpendicular to the spine neck axis, unless a cup-like shape would suggest another location of the synapse than on the tip of the head. Head volume was approximated by volume $={ }^{4} / 3 \pi$ (head radius $)^{2} *(0.5 *$ head length $)$. Neck length measurements are subject to a projection artifact caused by the relatively limited z-resolution $(\sim 600 \mathrm{~nm})$.

\section{FRAP experiments}

Bleaching was performed by line scanning the 2-photon beam for a period of $10 \mathrm{~ms}$ through the spine head, concomitant with $488 \mathrm{~nm}$ single-photon excitation line scanning to read out fluorescence levels in confocal mode. Bleaching power was around $5 \mathrm{~mW}$ in the back aperture of the objective. The bleaching period was preceded by a $0.5 \mathrm{~s}$ baseline scan, and followed by at least $3 \mathrm{~s}$ of scanning to measure recovery. The effective line scan frequency was $270 \mathrm{~Hz}$ with a 
pixel size of $35 \mathrm{~nm}$. For a subset of YFP spines, 2-photon mediated bleaching was performed as a discrete episode between baseline and recovery line scanning by scanning an area of $100 \mathrm{~nm}$ by $100 \mathrm{~nm}$ within the spine head of interest over approximately $300 \mathrm{~ms}$. For these experiments the bleaching power was reduced. The two bleaching schemes yielded identical results (linear regression $R^{2}=0.76$, fit slope 1.0; paired t-test $P=0.97 ; \mathrm{N}=14$ measurement pairs in 14 spines;

Suppl. Fig. 2). In all cases, the 2-photon laser power was adjusted so that bleaching levels were in the range of $25 \%$ to $75 \%$. Line scanning laser intensities were adjusted to give a stable baseline where confocal mode scanning itself did not induce bleaching. Fluorescence recovery was plotted as emission intensity versus time and fitted with a single-exponential function in ImageJ, wherefrom the recovery time constant, $\tau$, was derived ${ }^{9}$. For each spine 2 to 3 consecutive FRAP measurements were obtained and the average $\tau$ value used for further analysis, except for the depolarization experiments, where only one measurement was performed prior to the challenge. Alexa Fluor 488 (Invitrogen) was dissolved at $200 \mu \mathrm{M}$ in intra-cellular solution containing $125 \mathrm{mM}$ K-gluconate, $5 \mathrm{mM} \mathrm{KCl}, 10 \mathrm{mM}$ HEPES, 1 mM EGTA, 4 mM Mg-ATP, 0.3 mM Na-GTP, 10 mM Na-phosphocreatine. Cells not expressing YFP were voltage-clamped at $70 \mathrm{mV}$ in the whole-cell configuration, and loaded $5 \mathrm{~min}$ or more before experiments.

\section{Electrophysiology}

Whole-cell patch clamp recordings was performed using the intracellular solution described above, and glass pipettes with a tip resistance around $5 \mathrm{M} \Omega$. Depolarizations consisted of $4 \mathrm{~min}$ long voltage clamping to $0 \mathrm{mV}$ with $5 \mathrm{mM}$ QX-314 in the patch pipette. Consecutive STED images and FRAP measurements were obtained immediately before and around 2 minutes after 4 minutes of depolarization to $0 \mathrm{mV}$. Uncaging induced excitatory postsynaptic currents (uEPSCs) 
were recorded in the whole-cell mode with the addition of $5 \mu \mathrm{M} \beta$-actin in the intracellular solution, and with pipette tip resistances of around $10 \mathrm{M} \Omega$ to reduce intracellular dialysis ${ }^{19}$. Synaptic uncaging currents were measured at a membrane potential of $-70 \mathrm{mV}$. uEPSCs were averages of four repeats. Two uEPSC baseline time points were acquired in the presence of $\mathrm{MgCl}_{2}$, then $\mathrm{MgCl}_{2}$ was removed and $1 \mathrm{mM}$ tetrodotoxin (TTX) was added during LTP induction. After induction of LTP $\mathrm{MgCl}_{2}$ was added back to the solution and additional time points were acquired. Uncaging induced LTP (uLTP) was induced by uncaging glutamate at $0.5 \mathrm{~Hz}$ for 1 $\min ^{34}$. The caged glutamate compound was a modified version of the commercial MNIglutamate, which has a higher quantum efficiency (provided by Balázs Rózsa, Two-Photon Imaging Center, Institute of Experimental Medicine of the Hungarian Academy of Sciences). We used it at a concentration of $2 \mathrm{mM}$. The 2-photon laser was tuned to $740 \mathrm{~nm}$, and the pulses were $1 \mathrm{~ms}$ in length and delivered around $3 \mathrm{~mW}$ power into the back aperture of the objective. The uncaging pulses were controlled by a Pockels cell. For uncaging without electrophysiology, as in the uncaging plus STED imaging experiments, prior to experiments a cell in the slice culture was patch-clamped and the optimal uncaging power determined for the given conditions. The uncaging plus STED imaging experiments are therefore performed in unperturbed neurons.

During ULTP experiments STED images were acquired at $-5,1,15,30,45$ and 60 min relative to LTP induction. During LTP + FRAP experiments STED images were acquired at $-5,2,15$ and $30 \mathrm{~min}$ (the $1 \mathrm{~min}$ time point was not accessible due to the time required for switching from uncaging to FRAP wavelengths on the laser).

\section{Simulations of EPSPs}


EPSP amplitude as a function of $R_{\text {neck }}$ was calculated based on a simplified equivalent circuit of an electrically passive spine ${ }^{13}$. The model takes into account synaptic conductance $\left(G_{\text {syn }}\right)$, synaptic reversal potential $\left(E_{\mathrm{syn}}\right)$, spine neck resistance $\left(R_{\text {neck }}\right)$, and dendritic input resistance ( $\left.R_{\text {dend }}\right)$. With $R_{\text {spine }}=R_{\text {neck }}+R_{\text {dend }}$, the voltage in the spine head can be expressed as

$$
\operatorname{EPSP}_{\text {spine }}=\frac{G_{\text {syn }} * R_{\text {spine }} * E_{\text {syn }}}{1+G_{\text {syn }} * R_{\text {spine }}}
$$

and the voltage in the dendrite immediately below the spine neck as

$$
\operatorname{EPSP}_{\text {dend. }}=\frac{G_{\text {syn }} * R_{\text {dend }} * E_{\text {syn }}}{1+G_{\text {syn }} * R_{\text {spine }}}
$$

We set $R_{\text {dend }}$ to $50 \mathrm{M} \Omega$ and $E_{\text {syn }}$ to $+70 \mathrm{mV}$ (relative to the resting membrane potential of -70 $\mathrm{mV})^{36,57}$. Assuming $G_{\text {syn }}$ to be around $0.5 \mathrm{nS}^{57,58}$, we calculated EPSPs for three different values of $G_{\text {syn }}(0.1,0.5,2.5 \mathrm{nS})$, lumping together the contributions from AMPA and NMDA receptors. $R_{\text {dend }}$ is assumed constant after LTP and the model does not take into account any increases in $G_{\text {syn }}$ during $\mathrm{LTP}^{57}$. The effect of LTP on EPSPs thus reflects the drop by $50 \%$ in $R_{\text {neck }}$ after LTP.

\section{Data analysis}

No statistical methods were used to pre-determine sample sizes but our sample sizes are similar to, or exceed, those reported in previous publications ${ }^{6,9,25}$. Data collection and analysis were not performed blind to the conditions of the experiments. Normality of data distribution was tested by D'Agostino \& Pearson normality test. Data are presented as median values with inter-quartile range (IQR), or as mean with standard error mean (s.e.m.), standard deviation or the $95 \%$ 
confidence interval $(95 \% \mathrm{CI})$, as specified throughout. Statistical comparisons and parameters of variability were obtained using GraphPad software, as specified under Results. The level of significance was set to 0.05 and all tests are applied two-sided where applicable. In figures calculated probabilities are symbolized by asterisks as follows, ${ }^{*} p<0.05 ;{ }^{* *} p<0.01 ; * * * p<$ 0.001. Data were not randomized for analysis. Figure images are displayed as maximum intensity projections of z-stacks with a 1-pixel median filter and no additional processing. Lookup tables are Orange Hot or Fire (inverted). 


\section{REFERENCES}

1. Alvarez, V.A. \& Sabatini, B.L. Anatomical and physiological plasticity of dendritic spines. Annu Rev Neurosci 30, 79-97 (2007).

2. Holtmaat, A. \& Svoboda, K. Experience-dependent structural synaptic plasticity in the mammalian brain. Nat Rev Neurosci 10, 647-658 (2009).

3. Matsuzaki, M., et al. Dendritic spine geometry is critical for AMPA receptor expression in hippocampal CA1 pyramidal neurons. Nat Neurosci 4, 1086-1092 (2001).

4. Nusser, Z., et al. Cell type and pathway dependence of synaptic AMPA receptor number and variability in the hippocampus. Neuron 21, 545-559 (1998).

5. Lang, C., et al. Transient expansion of synaptically connected dendritic spines upon induction of hippocampal long-term potentiation. Proc Natl Acad Sci U S A 101, 16665-16670 (2004).

6. Matsuzaki, M., Honkura, N., Ellis-Davies, G.C. \& Kasai, H. Structural basis of long-term potentiation in single dendritic spines. Nature 429, 761-766 (2004).

7. Nägerl, U.V., Eberhorn, N., Cambridge, S.B. \& Bonhoeffer, T. Bidirectional activity-dependent morphological plasticity in hippocampal neurons. Neuron 44, 759-767 (2004).

8. Oh, W.C., Hill, T.C. \& Zito, K. Synapse-specific and size-dependent mechanisms of spine structural plasticity accompanying synaptic weakening. Proc Natl Acad Sci U S A 110, E305-312 (2013).

9. Svoboda, K., Tank, D.W. \& Denk, W. Direct measurement of coupling between dendritic spines and shafts. Science 272, 716-719 (1996).

10. Scheuss, V. \& Bonhoeffer, T. Function of Dendritic Spines on Hippocampal Inhibitory Neurons. Cereb Cortex (2013).

11. Majewska, A., Tashiro, A. \& Yuste, R. Regulation of spine calcium dynamics by rapid spine motility. J Neurosci 20, 8262-8268 (2000).

12. Araya, R., Jiang, J., Eisenthal, K.B. \& Yuste, R. The spine neck filters membrane potentials. Proc Natl Acad Sci U S A 103, 17961-17966 (2006).

13. Koch, C. \& Zador, A. The function of dendritic spines: devices subserving biochemical rather than electrical compartmentalization. J Neurosci 13, 413-422 (1993).

14. Palmer, L.M. \& Stuart, G.J. Membrane potential changes in dendritic spines during action potentials and synaptic input. J Neurosci 29, 6897-6903 (2009).

15. Chang, H.T. Cortical neurons with particular reference to the apical dendrites. Cold Spring Harb Symp Quant Biol 17, 189-202 (1952).

16. Crick, F. Do dendritic spines twitch? Trends in Neurosciences 5, 44-46 (1982).

17. Fifkova, E. \& Anderson, C.L. Stimulation-induced changes in dimensions of stalks of dendritic spines in the dentate molecular layer. Exp Neurol 74, 621-627 (1981).

18. Noguchi, J., Matsuzaki, M., Ellis-Davies, G.C. \& Kasai, H. Spine-neck geometry determines NMDA receptor-dependent Ca2+ signaling in dendrites. Neuron 46, 609-622 (2005).

19. Tanaka, J., et al. Protein synthesis and neurotrophin-dependent structural plasticity of single dendritic spines. Science 319, 1683-1687 (2008).

20. Urban, N.T., Willig, K.I., Hell, S.W. \& Nägerl, U.V. STED nanoscopy of actin dynamics in synapses deep inside living brain slices. Biophys J 101, 1277-1284 (2011).

21. Oray, S., Majewska, A. \& Sur, M. Effects of synaptic activity on dendritic spine motility of developing cortical layer v pyramidal neurons. Cereb Cortex 16, 730-741 (2006).

22. Parnass, Z., Tashiro, A. \& Yuste, R. Analysis of spine morphological plasticity in developing hippocampal pyramidal neurons. Hippocampus 10, 561-568 (2000).

23. Peebles, C.L., et al. Arc regulates spine morphology and maintains network stability in vivo. Proc Natl Acad Sci U S A 107, 18173-18178 (2010). 
24. Harris, K.M., Jensen, F.E. \& Tsao, B. Three-dimensional structure of dendritic spines and synapses in rat hippocampus (CA1) at postnatal day 15 and adult ages: implications for the maturation of synaptic physiology and long-term potentiation. J Neurosci 12, 2685-2705 (1992).

25. Harris, K.M. \& Stevens, J.K. Dendritic spines of CA 1 pyramidal cells in the rat hippocampus: serial electron microscopy with reference to their biophysical characteristics. J Neurosci 9, 2982-2997 (1989).

26. Heyman, N.S. \& Burt, J.M. Hindered diffusion through an aqueous pore describes invariant dye selectivity of Cx43 junctions. Biophys J 94, 840-854 (2008).

27. Kumar, M., Mommer, M.S. \& Sourjik, V. Mobility of cytoplasmic, membrane, and DNA-binding proteins in Escherichia coli. Biophys J 98, 552-559 (2010).

28. Einstein, A. Über die von der molekularkinetischen Theorie der Wärme geforderte Bewegung von in ruhenden Flüssigkeiten suspendierten Teilchen. Annalen der Physik 322, 549-560 (1905).

29. Petrasek, Z. \& Schwille, P. Precise measurement of diffusion coefficients using scanning fluorescence correlation spectroscopy. Biophys J 94, 1437-1448 (2008).

30. Alevra, M., Schwartz, P. \& Schild, D. Direct measurement of diffusion in olfactory cilia using a modified FRAP approach. PLoS One 7, e39628 (2012).

31. Major, G., Larkman, A.U., Jonas, P., Sakmann, B. \& Jack, J.J. Detailed passive cable models of whole-cell recorded CA3 pyramidal neurons in rat hippocampal slices. J Neurosci 14, 4613-4638 (1994).

32. Nevian, T., Larkum, M.E., Polsky, A. \& Schiller, J. Properties of basal dendrites of layer 5 pyramidal neurons: a direct patch-clamp recording study. Nat Neurosci 10, 206-214 (2007).

33. Grunditz, A., Holbro, N., Tian, L., Zuo, Y. \& Oertner, T.G. Spine neck plasticity controls postsynaptic calcium signals through electrical compartmentalization. J Neurosci 28, 13457-13466 (2008).

34. Harvey, C.D. \& Svoboda, K. Locally dynamic synaptic learning rules in pyramidal neuron dendrites. Nature 450, 1195-1200 (2007).

35. Bittner, K.C., Andrasfalvy, B.K. \& Magee, J.C. Ion channel gradients in the apical tuft region of CA1 pyramidal neurons. PLoS One 7, e46652 (2012).

36. Wong, R.K., Prince, D.A. \& Basbaum, A.I. Intradendritic recordings from hippocampal neurons. Proc Natl Acad Sci U S A 76, 986-990 (1979).

37. Tønnesen, J. \& Nägerl, U.V. Two-Color STED Imaging of Synapses in Living Brain Slices. Methods Mol Biol 950, 65-80 (2013).

38. Trommald, M. \& Hulleberg, G. Dimensions and density of dendritic spines from rat dentate granule cells based on reconstructions from serial electron micrographs. J Comp Neurol 377, 15-28 (1997).

39. Arellano, J.I., Benavides-Piccione, R., Defelipe, J. \& Yuste, R. Ultrastructure of dendritic spines: correlation between synaptic and spine morphologies. Front Neurosci 1, 131-143 (2007).

40. Jones, E.G. \& Powell, T.P. Morphological variations in the dendritic spines of the neocortex. J Cell Sci 5, 509-529 (1969).

41. Peters, A. \& Kaiserman-Abramof, I.R. The small pyramidal neuron of the rat cerebral cortex. The perikaryon, dendrites and spines. Am J Anat 127, 321-355 (1970).

42. Spacek, J. \& Hartmann, M. Three-dimensional analysis of dendritic spines. I. Quantitative observations related to dendritic spine and synaptic morphology in cerebral and cerebellar cortices. Anat Embryol (Berl) 167, 289-310 (1983).

43. Stewart, M.G., et al. Chemically induced long-term potentiation increases the number of perforated and complex postsynaptic densities but does not alter dendritic spine volume in CA1 of adult mouse hippocampal slices. Eur J Neurosci 21, 3368-3378 (2005).

44. Harnett, M.T., Makara, J.K., Spruston, N., Kath, W.L. \& Magee, J.C. Synaptic amplification by dendritic spines enhances input cooperativity. Nature 491, 599-602 (2012). 
45. Yuste, R. \& Bonhoeffer, T. Morphological changes in dendritic spines associated with long-term synaptic plasticity. Annu Rev Neurosci 24, 1071-1089 (2001).

46. Fonseca, R., Nagerl, U.V., Morris, R.G. \& Bonhoeffer, T. Competing for memory: hippocampal LTP under regimes of reduced protein synthesis. Neuron 44, 1011-1020 (2004).

47. Yuste, R. Electrical compartmentalization in dendritic spines. Annu Rev Neurosci 36, 429-449 (2013).

48. Wilson, C.J. Passive cable properties of dendritic spines and spiny neurons. J Neurosci 4, 281-297 (1984).

49. Gulledge, A.T., Carnevale, N.T. \& Stuart, G.J. Electrical advantages of dendritic spines. PLoS One 7, e36007 (2012).

50. Gähwiler, B.H. Slice cultures of cerebellar, hippocampal and hypothalamic tissue. Experientia 40, 235-243 (1984).

51. Nägerl, U.V., Willig, K.I., Hein, B., Hell, S.W. \& Bonhoeffer, T. Live-cell imaging of dendritic spines by STED microscopy. Proc Natl Acad Sci U S A 105, 18982-18987 (2008).

52. Hell, S.W. \& Wichmann, J. Breaking the diffraction resolution limit by stimulated emission: stimulated-emission-depletion fluorescence microscopy. Opt Lett 19, 780-782 (1994).

53. Klar, T.A., Jakobs, S., Dyba, M., Egner, A. \& Hell, S.W. Fluorescence microscopy with diffraction resolution barrier broken by stimulated emission. Proc Natl Acad Sci U S A 97, 8206-8210 (2000).

54. Imspector. www.max-planck-innovation.de/de/industrie/technologieangebote/software/.

55. Urban, N.T., Willig, K.I., Hell, S.W. \& Nagerl, U.V. STED Nanoscopy of Actin Dynamics in Synapses Deep Inside Living Brain Slices. Biophys J 101, 1277-1284 (2011).

56. Tønnesen, J., Nadrigny, F., Willig, K.I., Wedlich-Soldner, R. \& Nägerl, U.V. Two-Color STED Microscopy of Living Synapses Using A Single Laser-Beam Pair. Biophys J 101, 2545-2552 (2011).

57. Benke, T.A., Luthi, A., Isaac, J.T. \& Collingridge, G.L. Modulation of AMPA receptor unitary conductance by synaptic activity. Nature 393, 793-797 (1998).

58. Gasparini, S., Migliore, M. \& Magee, J.C. On the initiation and propagation of dendritic spikes in CA1 pyramidal neurons. J Neurosci 24, 11046-11056 (2004). 


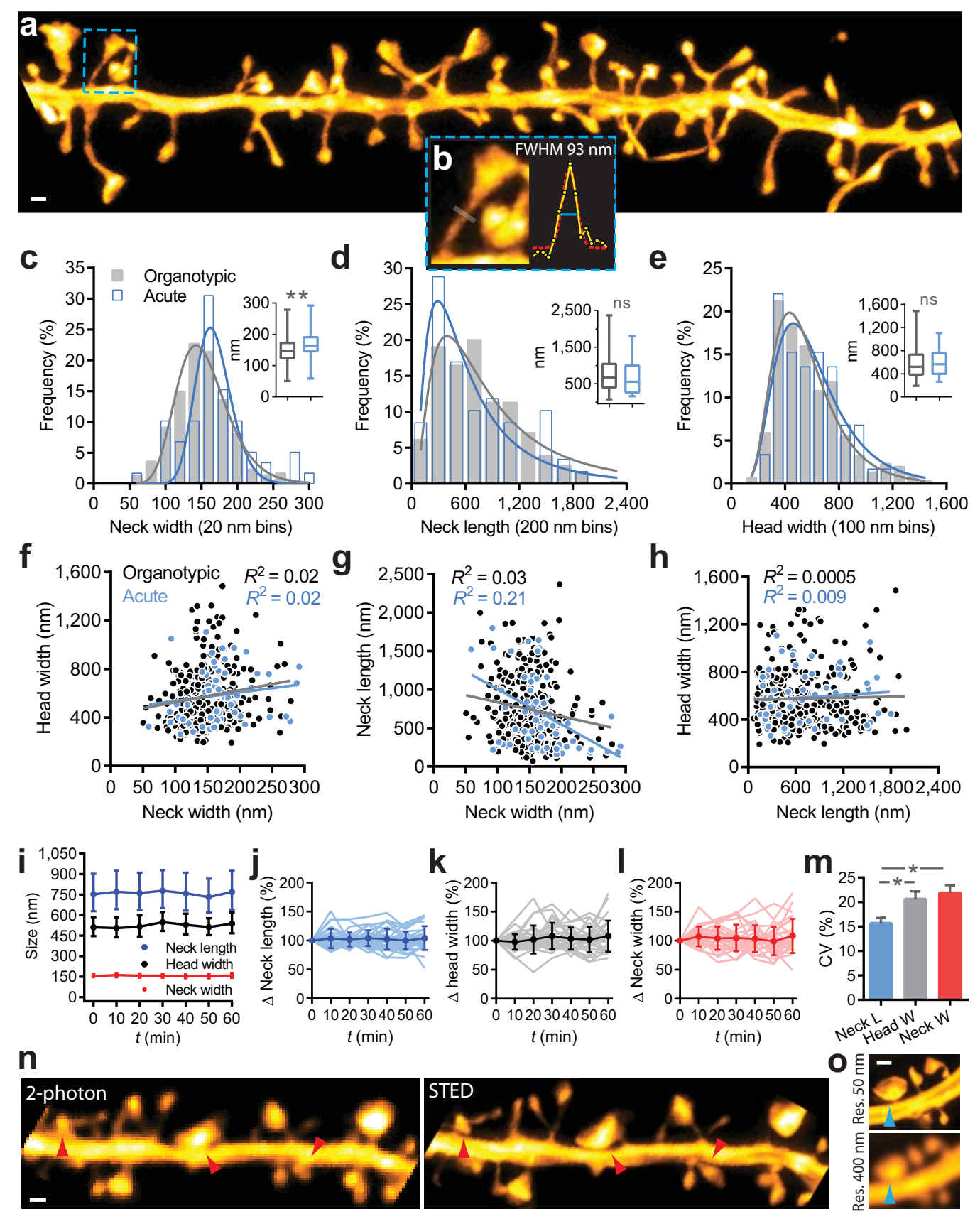




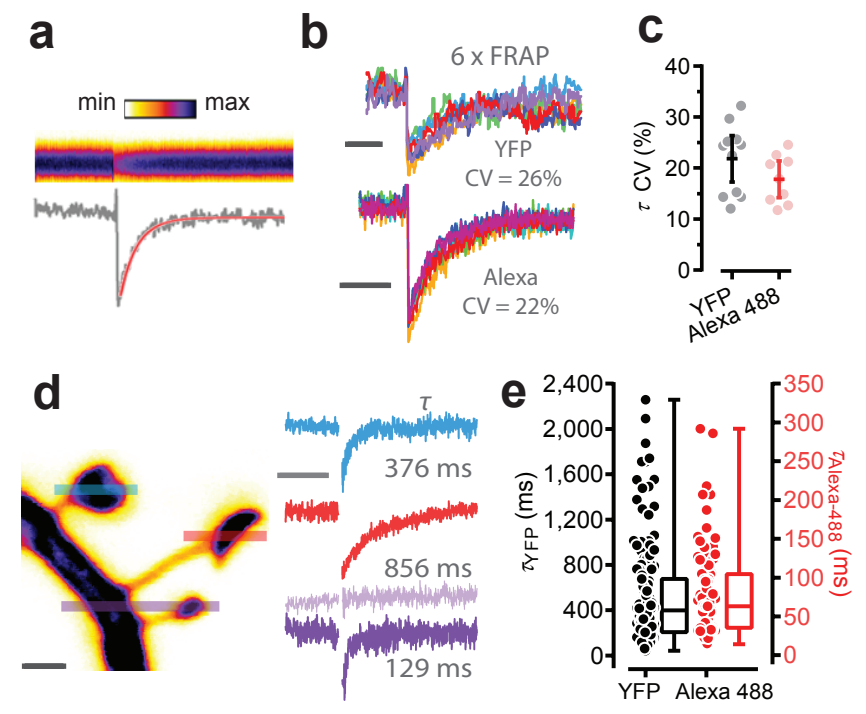



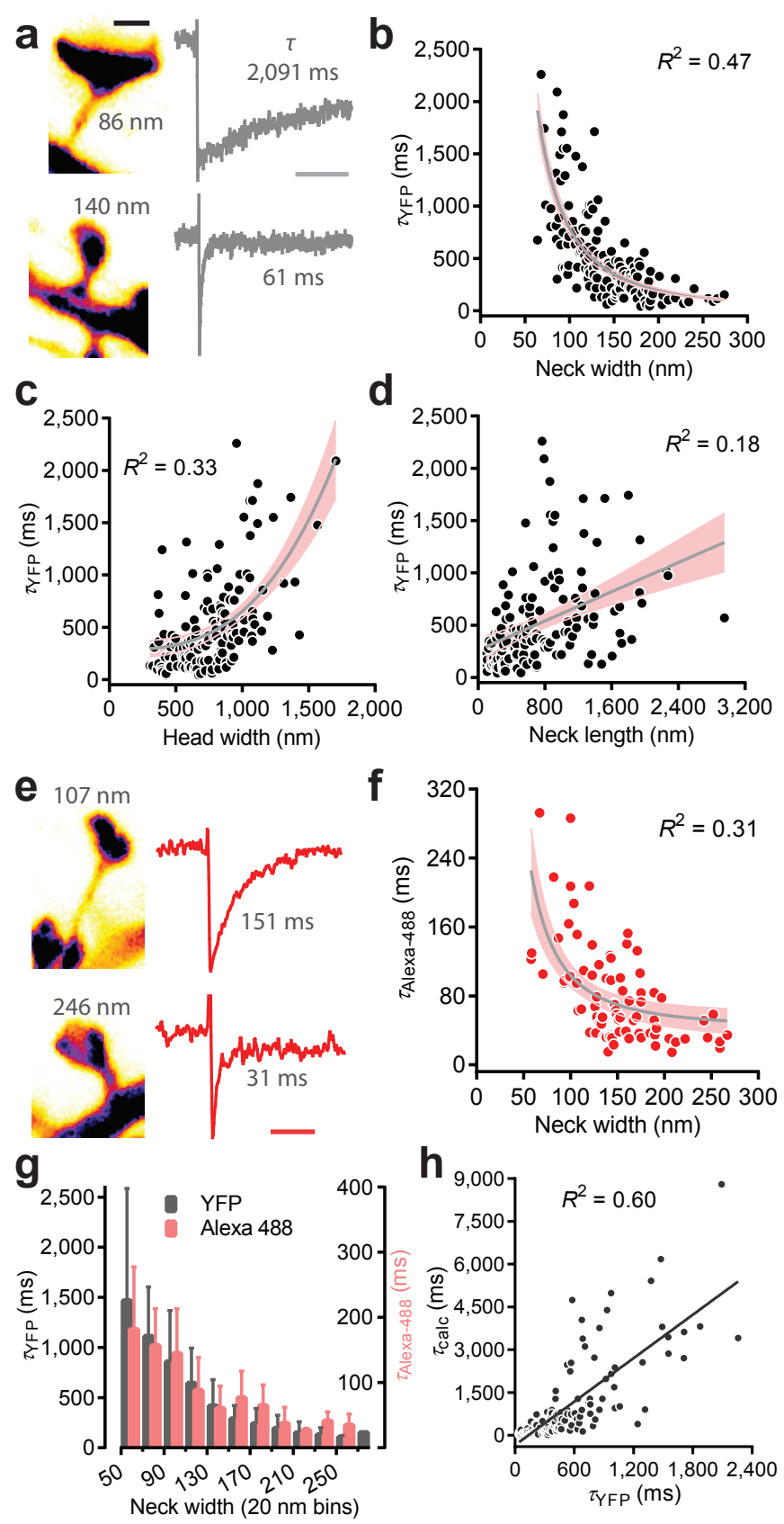
a

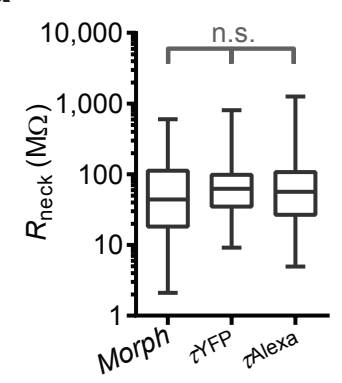

C

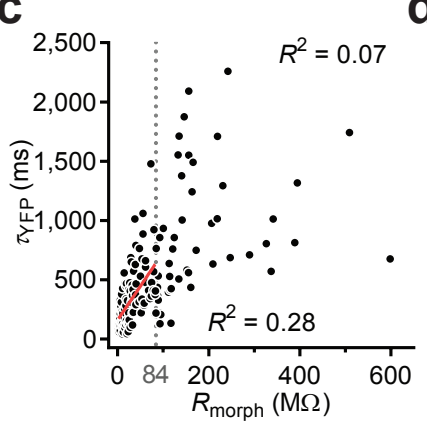

b

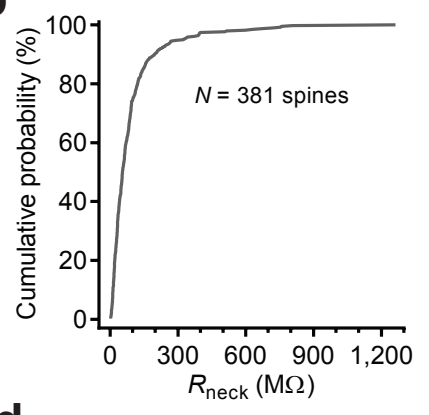

d

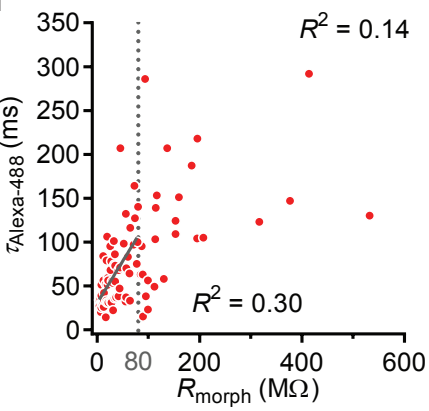



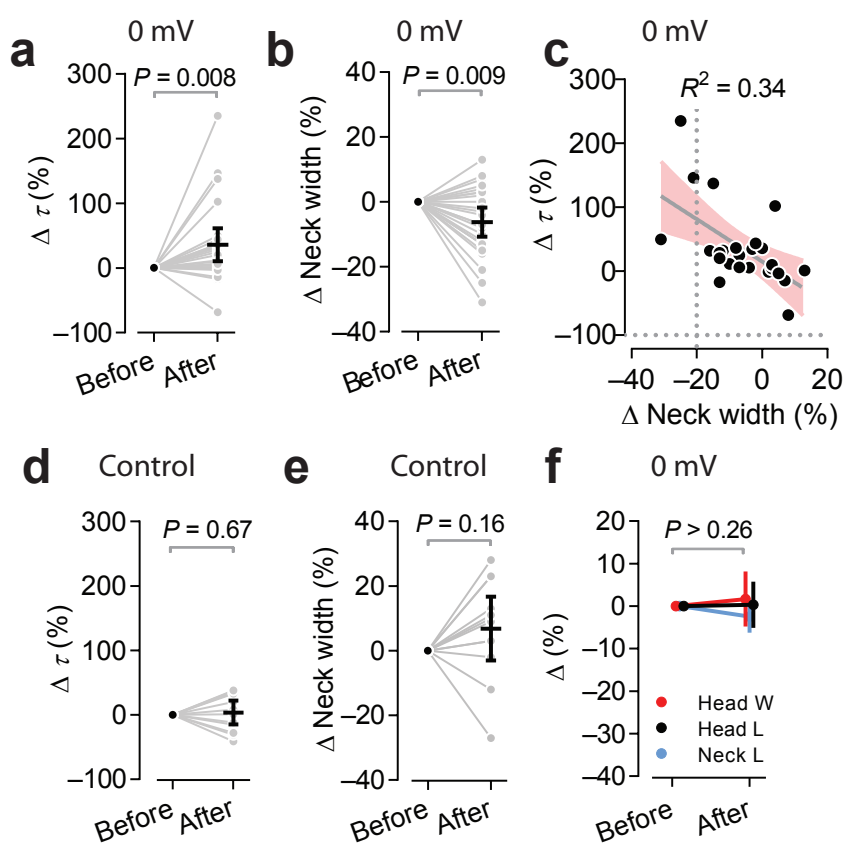


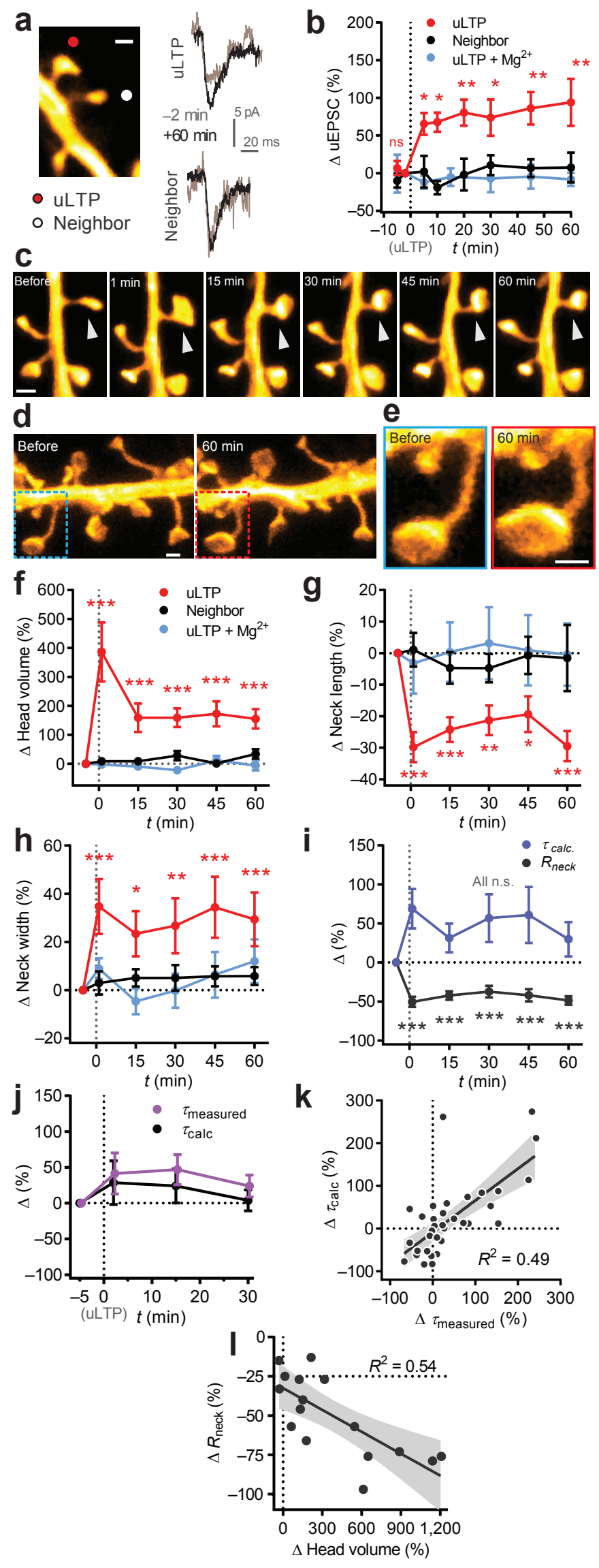



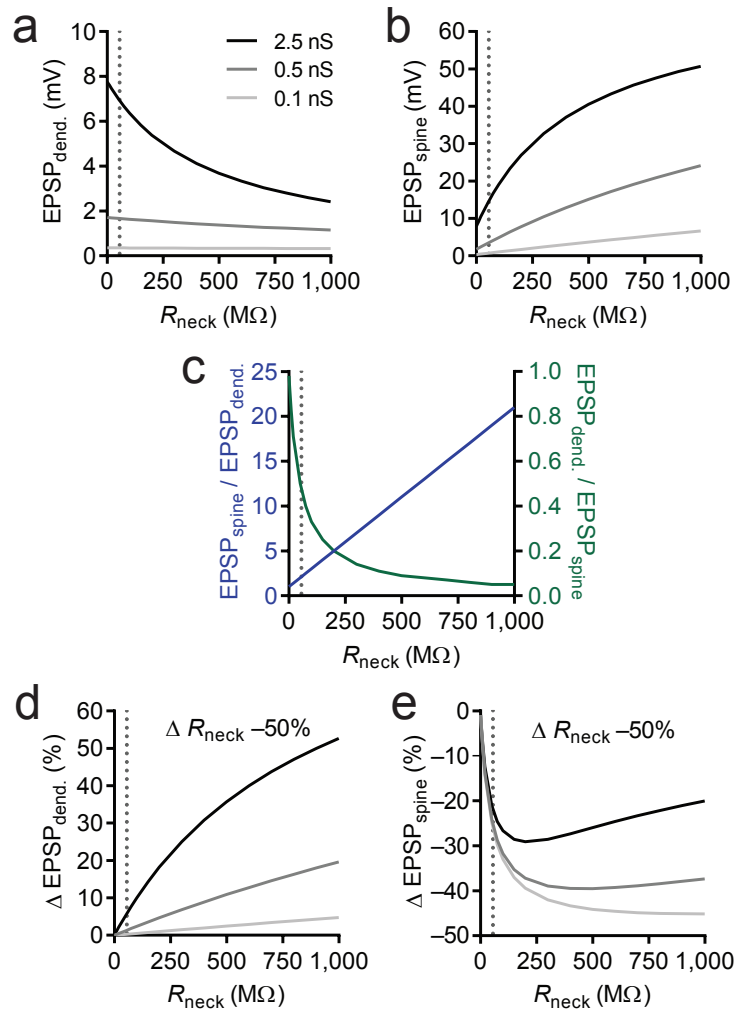